\title{
ON THE DAMAGE TO METALLIZED FILM CAPACITORS CAUSED BY ELECTRICAL DISCHARGES
}

\author{
J. W. BURGESS, R. T. BILSON and N. F. JACKSON \\ Allen Clark Research Centre, The Plessey Company Limited, Caswell, Towcester, Northants, U.K.
}

(Received April 23, 1975; in final form June 20,1975)

\begin{abstract}
High voltage operation of metallized film capacitors leads to electrical spark breakdown in pockets of gas trapped within the sealed capacitor unit. A simple picture of gas discharge processes in the capacitor is used to identify the role played by electric discharges in the deterioration and ultimate failure of capacitors under accelerated life test.
\end{abstract}

\section{INTRODUCTION}

The development of metallized solid polymer film capacitors for use at high voltage requires considerable care with the processing technology. In the absence of liquid impregnants, the application of peak voltages above $300 \mathrm{~V}$ across the capacitor can result in electrical spark breakdown being initiated in pockets of air trapped within the sealed unit, causing localized damage to the polymer film and the metallization, and a considerable generation of heat. In the present paper, the occurrence of breakdown resulting from gas discharges in wound capacitor units of the extended foil construction ${ }^{1}$ is discussed. The polymer film is metallized on one side and wound in a staggered configuration allowing the whole length of metallized electrode to be short circuited to an end connection in the form of a fine spray (Figure 1). Using simple physical considerations, a picture of the damage caused by electrical breakdown and the subsequent deterioration in capacitor performance may be developed.

\section{INITIATION OF SPARK BREAKDOWN}

When the electric stress through a gas is increased to exceed some critical value, a spark breakdown will initiate a momentary discharge in the gas. The critical stress is known as the Discharge Inception Stress $\left(E_{i}\right)$ and is dependent on gas composition, temperature $(\mathrm{T})$ and pressure $(\mathrm{P})$. The voltage associated with path of length $\mathrm{d}^{\prime}$ over which a uniform critical stress is maintained, is the Discharge Inception Voltage $\left(V_{i}\right)$. Over a wide range of conditions, the Discharge
Inception Voltage is found to be a function of the product of gap $\left(d^{\prime}\right)$ and pressure $(P)$, the relationship being known as Paschen's Law,

$$
V_{i}=f\left(P \cdot d^{\prime}\right)
$$

(a) Wound unit

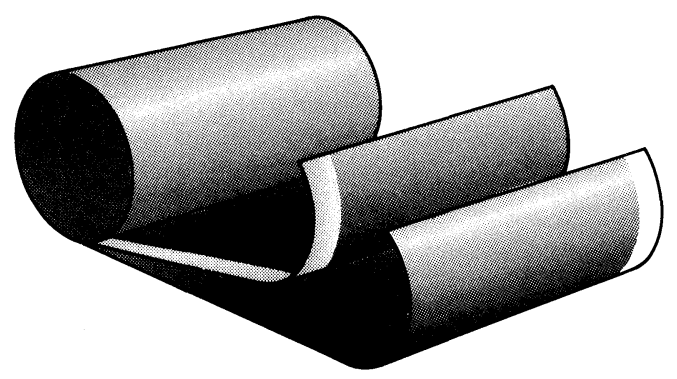

(b) Section through wound unit

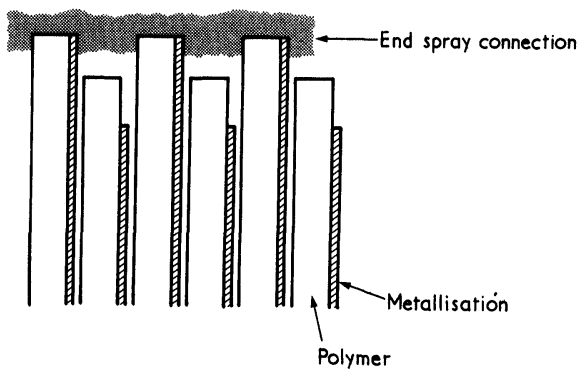

FIGURE 1 Extended foil configuration: (a) Wound Unit, (b) Section through wound unit. 


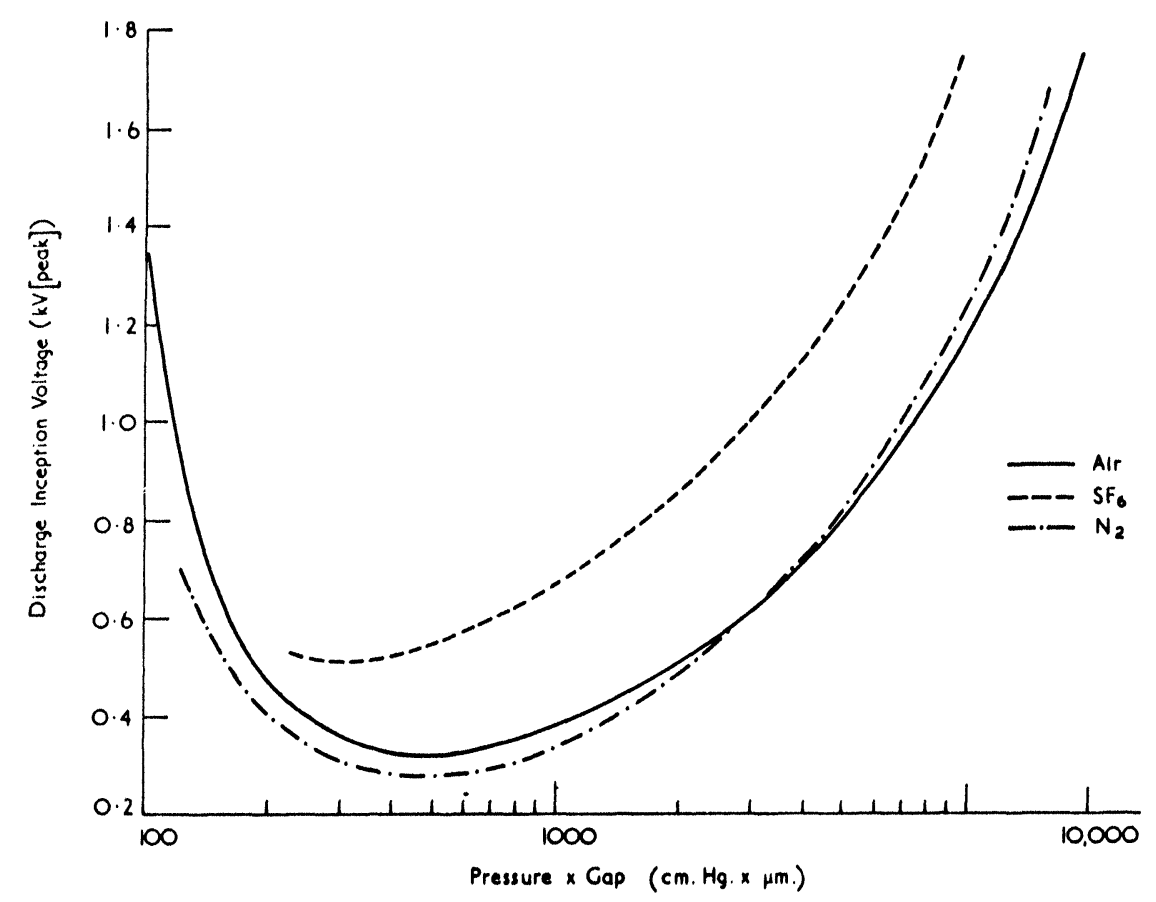

FIGURE 2 Discharge inception voltage for air, $\mathrm{N}_{2}$ and $\mathrm{SF}_{6}$ at $20^{\circ} \mathrm{C}$ versus pressure and gap.

In Figure 2 Paschen plots for air, nitrogen and sulphur hexafluoride at $20^{\circ} \mathrm{C}$ are shown ${ }^{2}$. The rise in $\mathrm{V}_{\mathrm{i}}$ for small pressure and gap is a consequence of insufficierit molecules for the usual avalanche of ionization to be produced. This relationship results in a minimum value of Inception Voltage which, for pressures near atmospheric, corresponds to a breakdown path length of $10 \mu \mathrm{m}$. The nature of the solid interfaces from which eletric stress is applied has a limited effect on the inception voltage, but can alter the discharge characteristics. For example, in the case ofdischarges across a gaseous cavity within a dielectric solid, the discharge magnitude is limited by the impedance of the dielectric and the repetition frequency is determined by the time taken for charge leakage to restore the electric stress across a previously discharged path (typically $10^{2} \mathrm{sec}$. in plastic material). Breakdown will occur along paths of minimum inception voltage, of which there may be several associated with a single cavity. Repeated discharging to a local site on the boundary of a cavity eventually causes considerable damage to the dielectric. Under a.c. applied stress the frequency of repetition for discharges is equal to twice the signal frequency ${ }^{3}$, and can be some orders of magnitude higher than that for the d.c. case, resulting in an acceleration of damage caused to the dielectric.
Similar breakdown characteristics are observed for discharges in a gas space between the surface of a dielectric material and an external conductor. In this case the local discharges are still repeated every halfcycle of the a.c. signal, but the discharge magnitude increases rapidly as the voltage is increased above $V_{i}$.

A significant volume of gas is contained in the wound capacitor unit principally near the staggered non-contact edge of the polymer film (Figure 1), but also trapped within the windings. Some gas remains after the conventional heat treatment and further gas pockets are contained within the polymer film, usually associated with inclusions formed during the casting of the film, and known as voids (Figure 3). When the capacitor is operated at high voltage, trapped gas pockets are susceptible to breakdown with consequent damage to the polymer film and aluminium metallization. The initiation of discharges in the capacitor is thought to be governed by the general theory of gas breakdown, the discharge gaps being typically $<10 \mu \mathrm{m}$. Some deviations from Paschen's Law have been observed with small gaps $(<5 \mu \mathrm{m})$, the inception voltage always being lower than the Paschen value (4), but not below the characteristic Paschen minimum. Typical values of uniform electric stress developed in working capacitors do not exceed thresholds for field emission of 
electrons from the metallization, and consequently severe departures from Paschen's Law should not occur frequently. The a.c. operation serves to mask polarity effects, which are not discussed.

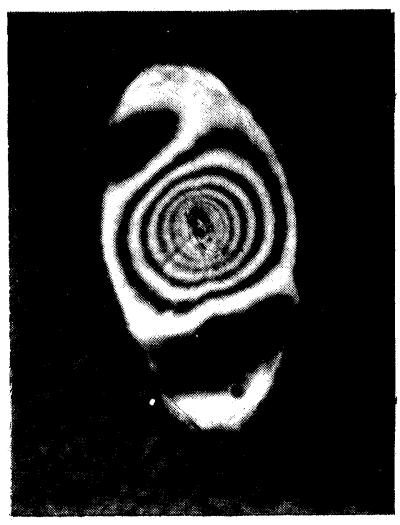

FIGURE 3 Void in the film as viewed with reflected light. Typical void densities are $\sim 25$ to $100 \mathrm{~cm}^{-2}$.

\subsection{Cavity Discharge}

In Figure 4(a), two types of gaseous cavity which might lie within the wound unit are shown in section. The sketch illustrates voids within the polymer film and localized gas pockets trapped within the windings. The standard winding gap is assumed to be $<0.5 \mu \mathrm{m}$, with breakdown being localized at trapped gas pockets. In each case, the cavity is roughly disc-shaped and minimally disturbs the uniform electric field pattern between the aluminium electodes. The concentration of electric stress at regions of lower dielectric constant results in the Discharge Inception Voltage for cavity breakdown (3) being

$$
\mathrm{V}_{\mathrm{i}}=\mathrm{V}_{\mathrm{g}}\left(1+\left(\frac{\mathrm{d}}{\mathrm{d}^{\prime}}-1\right) \frac{1}{\epsilon}\right)
$$

where $\mathrm{V}_{\mathrm{g}}$ is the gas breakdown voltage, $\mathrm{d}$ is the distance between adjacent electrodes, $\mathrm{d}^{\prime}$ is the discharge gap (Figure 4(a)), and $\epsilon$ is the dielectric constant of the polymer. Values of $V_{i}$ as a function of gas path length are shown in Figure $4(\mathrm{~b})$ for various pressures within the cavity for $8 \mu \mathrm{m}$ thick polymer film in air at $20^{\circ} \mathrm{C}$. The gas breakdown voltage $\mathrm{V}_{\mathrm{g}}$ is taken as the Paschen value appropriate to $\mathrm{d}^{\prime}$ and the polymer dielectric constant is taken as 2.3 . (a) Cavity Model

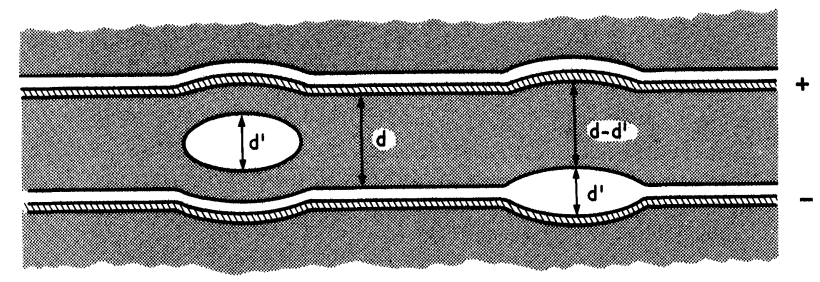

(b) Cavity Discharge Inception Voltage Versus Gap for Various Pressures

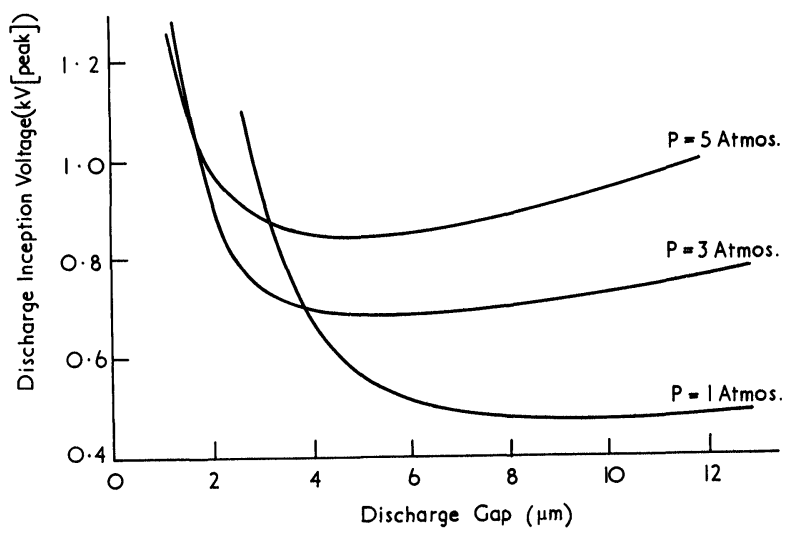

FIGURE 4 Cavity discharges: (a) Cavity model, (b) Cavity discharge inception voltage versus gap for various pressures.

\subsection{Conductor Edge Discharge}

The extended foil configuration utilizes staggered alternate polymer films, for which the non-contact aluminium electrode is recessed. The enhancement of electric field near the edge of the aluminium electrode causes this region to be highly vulnerable to discharges. Assuming a uniform field across the gas path $\mathrm{d}^{\prime}$ shown in Figure 5(a), the Discharge Inception Voltage $V_{i}$ for conductor edge breakdown (3) may be determined as

$$
\mathrm{V}_{\mathrm{i}}=\mathrm{V}_{\mathrm{g}}\left(1+\frac{\mathrm{d}}{\mathrm{d}^{\prime} \cdot \epsilon}\right)
$$

where $d$ is the thickness of the polymer film. In Figure 5(b), values of $V_{i}$ are shown as a function of gas path length $\mathrm{d}^{\prime}$ for various pressures, again wth $8 \mu \mathrm{m}$ thick polymer film in air at $20^{\circ} \mathrm{C}$.

In a typical capacitor unit, the value of $V_{i}$ for the conductor edge only loosely corresponds to that calculated from Eq. 3, because of the strong non- 
(a) Conductor Edge Model

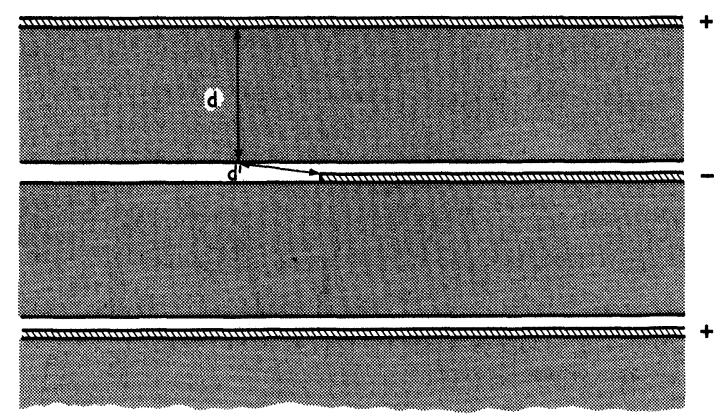

(b) Conductor Edge Discharge Inception Voltage Versus Gap for Various Pressures

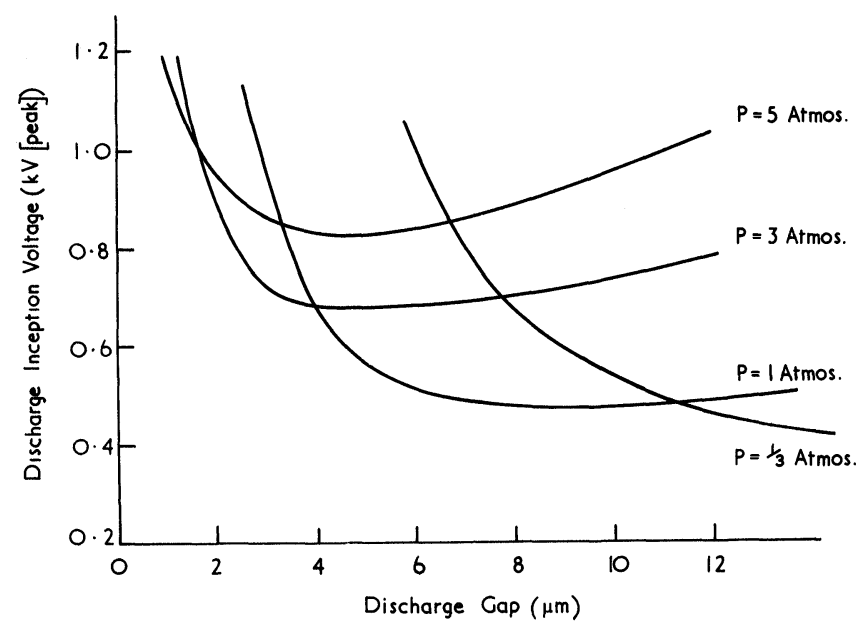

FIGURE 5 Conductor edge discharges: (a) Conductor edge model, (b) Conductor edge discharge inception voltage versus gap for various pressures.

uniformity of electric field in the gas. This electric field patern close to the electrode edge has been calculated for the geometry shown in Figure 6 by standard mapping techniques ${ }^{5}$. The average winding gap is taken to be $0.5 \mu \mathrm{m}$ (this value is not critical to the electric field behaviour) and the electrode thickness to be $300 \AA$. A contour representation of the electric field is shown, where the field magnitude is normalized to the uniform field $\mathrm{E}_{0}$ across the polymer $(\epsilon=2.3, \mathrm{~d}=8 \mu \mathrm{m})$

$$
E_{0}=\frac{V}{d}
$$

It is seen from Figure 6 that $\mathrm{E}>\mathrm{E}_{0}$ for distances $<2 \mu \mathrm{m}$ from the conductor edge. Corona streamers are readily initiated within the high field region and will propagate into regions of low stress, some of them reaching the polymer surface and causing complete breakdown of the gas. The propagation of streamers once formed has been observed to continue in regions of quite low field strength ${ }^{6}$. Overall discharge characteristics are quite complex, being critically dependent on the polarity of the conductor edge, the composition of gas and ambient conditions.

\section{DETERIORATION OF CAPACITOR PERFORMANCE}

The simple models developed here are considered representative of the types of gas breakdown occurring in a metallized film capacitor. A similar representative approach can be used to assess the impact of gas discharges on the performance of a typical capacitor. In the cases shown in Figures 4(a) and 5(a) surface damage arising from localized discharges may be expected on both internal and external surfaces of the polymer and on the aluminium electrodes. For high applied voltage $(>500 \mathrm{~V})$ the discharge magnitude and consequent damage increases more for the aluminium $\leftrightarrow$ polymer discharges, than for those occurring between two polymer surfaces ${ }^{7}$.

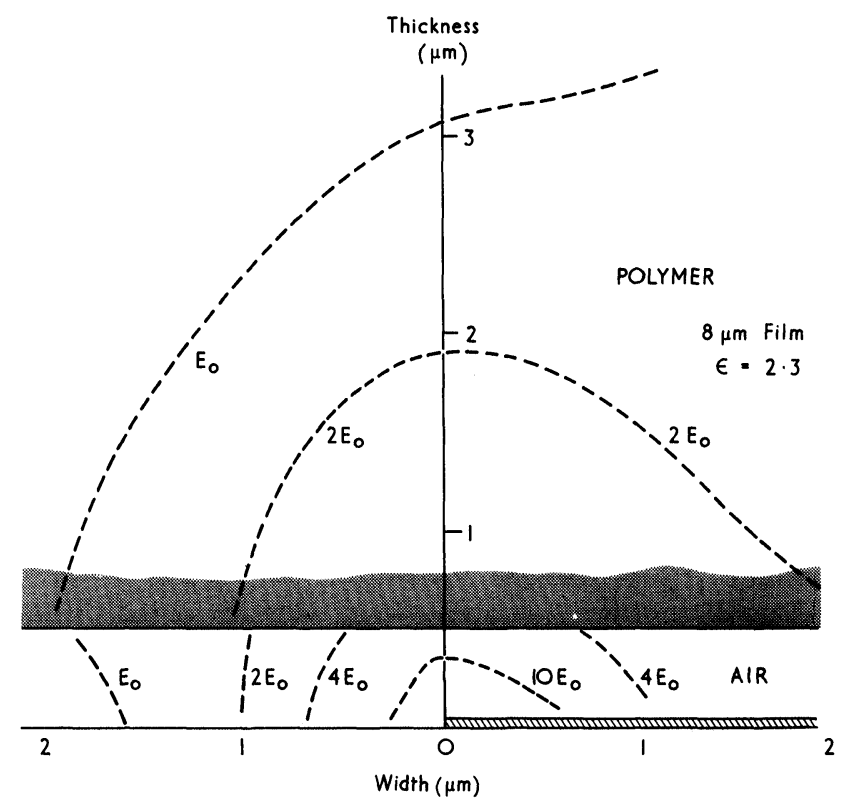

FIGURE 6 Electric field distribution near conductor edge relative to uniform field, $\mathrm{E}_{0}$. 
The damage to polymer and electrode surfaces may be chemical, via active gases formed within a discharge, or physical due to bombardment by charge carriers. A discharge may be summarily described in terms of electrons accelerated under the applied field, colliding with and ionizing neutral gas molecules when sufficient energy has been gained. A typical energy for charge carriers arriving at a solid surface is then $\sim 8 \mathrm{eV}$ (half of the ionization potential of the gas). With polymeric bond energies being $\sim 4 \mathrm{eV}$, considerable chemical modifications in the polymer will occur near a discharge site. Observed discharge magnitudes are $\sim 1$ to $1000 \mathrm{pC}$, and the associated energy can produce very high local surface temperatures. A single discharge of $10 \mathrm{pC}$ produces $10^{-11}$ calories at the electrode surface (assuming $8 \mathrm{eV}$ energy on arrival) which is sufficient to sublime $0.25 \mu \mathrm{m}$ square area of the aluminium electrode. The erosion of internal polymer surfaces by discharges has been observed ${ }^{7}$ and amounts of displaced polymer are similar to those estimated for the aluminium electrode.

The effects of repeated discharging thus causes some chemical modification of the polymer surface, erosion of the polymer and eventual formation of breakdown channels by treeing ${ }^{8}$, and also erosion of the aluminium electrode. Evidence for chemical damage in the present work was obtained by infra-red spectroscopy which indicated the presence of carbonyl groups. Local damage to the polymer film has little effect on capacitor performance until a complete breakdown channel through the film is developed. Complete sublimation of the aluminium at discharge sites leads to a loss of electrode area and proportional loss of capacitance. The removal of aluminium, together with modifications to capacitor geometry caused by heat generated in the discharges are major contributors to the loss of capacitance and final catastrophic breakdown of the devices.

From the representative models considered, two contributions to the capacitance loss under life test may be identified.

1) Repeated discharges across gas pockets trapped within the windings lead directly to removal of the aluminium electrode. Once a small area of electrode has been removed, further discharges will occur from each point on the periphery of the surrounding aluminium electrode via the conductor edge mechanism, leading to the growth of large circular clearance areas in the aluminium.

2) At the staggered non-contact edge of the aluminium electrode, discharges occurring via the
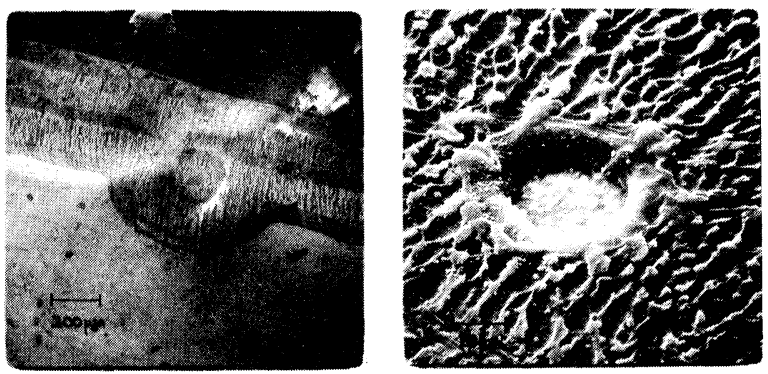

FIGURE 7 (a) Erosion of metallisation, illustrating overlap of edge erosion and circular clearance, (b) Erosion of polymer film.

conductor edge mechanism will cause the electrode to recede from its initial position, at a rate uniform throughout the length of the film.

Both of these characteristic modifications are observed in practical life tested capacitors (Figure 7). The circular clearance areas are found to lie at random positions, while the erosion of the noncontact electrode edge is uniform along the length of film. Only an occasional correlation is found between the electrode clearance areas and the sites of voids within the polymer itself. A study of life-tested capacitors has shown that small areas of the aluminium electrode can be sublimed from the polymer film, especially in the initial stages of life testing. As the life test proceeds, further electrode removal is effected by the oxidation of aluminium. The rate of electrode removal is not constant with time, owing to changes in the ambient conditions for discharging. Heat dissipation within the unit is fairly efficient, but a general temperature rise in the body of the capacitor is observed, and this will lead to a reduction in the Paschen voltage of the gas. The gas pressure and composition are also continually changing, a sealed air-filled capacitor often showing firstly a slight drop in pressure associated with the using up of oxygen, and later a sharp increase in pressure caused by copious generation of hydrogen gas.

Quantitative predictions of deterioration in a thin film capacitor unit are difficult, owing to uncertainties about the ambient conditions at a local discharge site and the changes which occur after discharges have commenced. However, the simple models used here identify the basic processes involved in capacitor deterioration, and may be used to provide a semiquantitative assessment of the performance to be anticipated from particular devices. Within the capacitor unit are many possible discharge paths, 


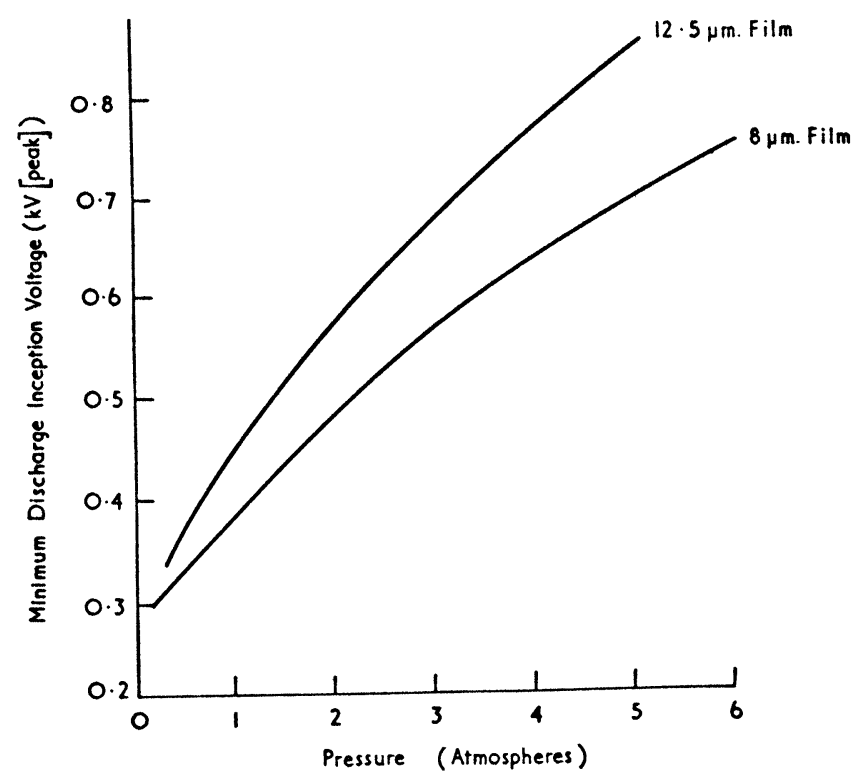

FIGURE 8 Minimum discharge inception voltage versus pressure.

varying from $0.1 \mu \mathrm{m}$ to $\sim 5 \mu \mathrm{m}$ in length, and extending considerably further when the conductor edge mechanism (Figure 5(a)) is invoked. If a continuous distribution of path lengths is available, then the characteristic Discharge Inception Voltage for the two representative models will be given simply by the minima of the curves plotted in Figures 4(b) and 5(b). The resulting variation of Discharge Inception Voltage with pressure for $8 \mu \mathrm{m}$ and $12 \mu \mathrm{m}$ film is shown in Figure 8, taking the conductor edge model as an example. Thus an increase in pressure will, under the present assumptions, raise the Discharge Inception Voltage and, for a given test voltage, reduce the number of discharges. Reductions in the numbers of discharges may also be obtained by varying the gas composition within the capacitor, in particular using electro-negative gases such as $\mathrm{SF}_{6}$. Figure 2 shows the measure of increase in Paschen voltage (and therefore of Discharge Inception Voltage) obtained with the use of pure $\mathrm{SF}_{6}$. A nitrogen atmosphere is sometimes preferred in capacitor design, since although there is little increase in Paschen voltage over that of air, the oxygen required for oxidation of the aluminium is limited to that available in residual moisture, and the rate of capacitance loss can be slowed down.

To illustrate the use of discharge properties in the development of capacitor design, a set of life tests on several different types of capacitor has been analysed. The capacitance of the units was recorded at various times within the duration of the tests, and the analysis was made using a procedure analogous to that employed for the assessment of bulk insulating materials 9 . The minimum discharge inception voltage is calculated from a knowledge of capacitor construction and the ambient conditions, and the ratio of test voltage to inception voltage $\left(\mathrm{V} / \mathrm{V}_{\mathrm{I}}\right)$ is plotted against the number of continuous a.c. cycles producing a capacitance drop of 3\% (Figure 9). Having taken the varying ambient conditions into account in the calculation of inception voltage, the results fall into two groups, according to the types of encapsulation used. In this way, the techniques developed here can be used to identify and isolate the effects of particular design variations on capacitor performance.

The results for each group of capacitors follow the characteristic form for lifetime of plastic materials,

$$
\mathrm{N}=\mathrm{K}\left(\frac{\mathrm{V}_{\mathrm{i}}}{\mathrm{V}}\right)^{\mathrm{n}}
$$

where $\mathrm{N}$ is the number of cycles, $\mathrm{K}$ is a constant and the value of the index $n$ is found from Figure 9 to be $\mathrm{n} \sim 8$. This value agrees with results previously obtained for a number of plastic bulk materials 9 subjected to internal gas discharges.

\section{CAPACITOR BREAKDOWN}

As the life test proceeds, the deterioration of the device continues with further electrode removal and generation of heat until a complete failure eventually occurs. Three possible failure mechanisms resulting from the occurrence of gas discharges are:

1) Breakdown of the polymer film by thermal runaway or electromechanical effects.

2) The completion of a breakdown channel through the polymer film.

3) Damage to the sprayed end-connections leading to an open circuited device.

It is thought that for typical polymer films, electromechanical breakdown will occur before a thermal runaway condition develops ${ }^{3}$. The strong electric field applied across the polymer is accompanied by a compressive stress, causing a distortion of the film. In regions close to local discharge sites, the temperature of the polymer is considerably increased, and the accompanying decrease of elastic stiffness leads to a mechanical collapse of the film. The erosion of polymer caused by repeated discharges will 


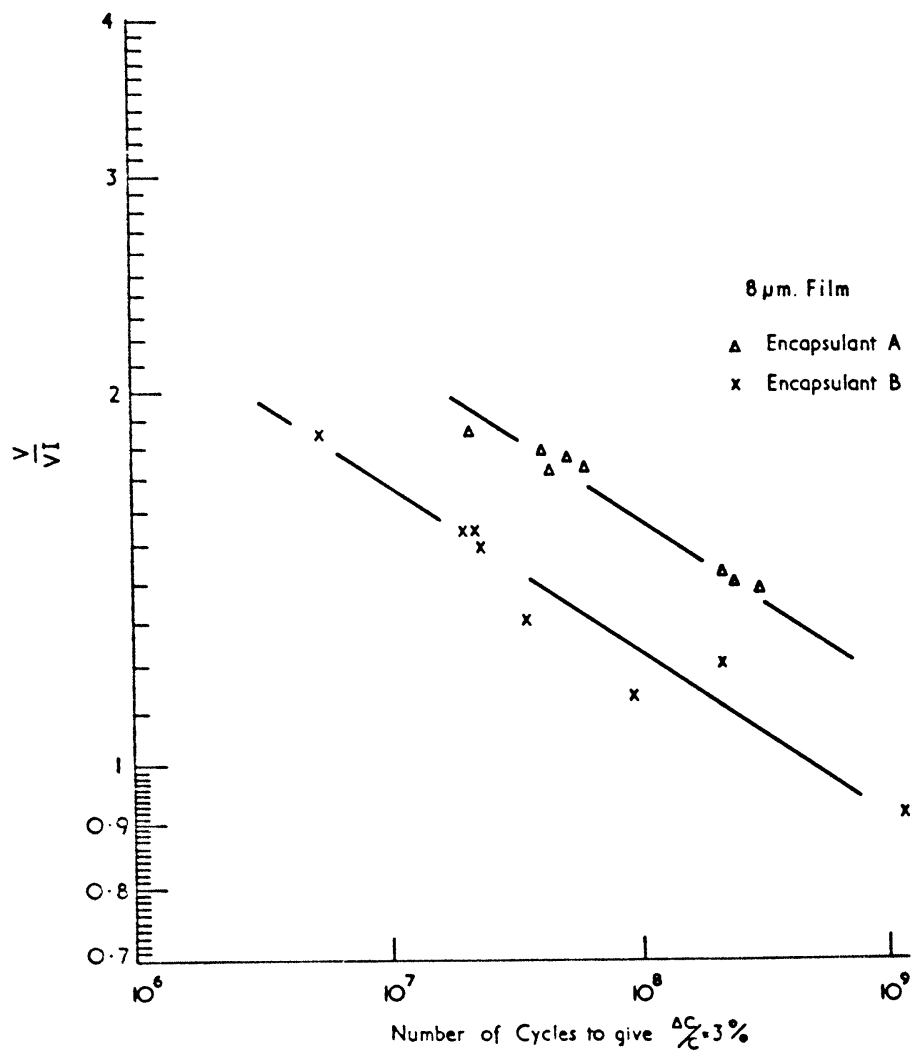

FIGURE 9 Ratio of test voltage to discharge inception voltage versus number of a.c. test cycles to give a $3 \%$ capacitance loss.

form discharge channels which eventually propagate through the film, precipitating failure by mechanism (2). The distortion of the film during life test induces stress at the sprayed end-connection, and damage to the contact with the aluminium electrode is intensified with the disruption of the dissipative flow of heat out of the capacitor. Failure by mechanism (3) can be a common problem with the extended foil configuration.

\section{CONCLUSIONS}

A quantitative picture has been given of some processes of deterioration in metallised film capacitors under life test at high voltage. Much of the structural damage to the polymer and electrodes can be associated with gas discharges within the wound unit. The deterioration in capacitor performance has been analysed in terms of discharge characteristics for two types of gaseous pockets occurring in the extended foil configuration for a capacitor. The predicted behaviour has similar characteristics to the aging of bulk plastic materials by discharges in gas filled cavities within the plastic. Observed rates of deterioration for life-tested capacitors under various ambient conditions conform with predicted rates, so establishing structural damage from discharges as a primary contributor to capacitor deterioration and failure.

\section{ACKNOWLEDGEMENTS}

The authors are grateful to Drs. J. C. Bass, P. J. Harrop and A. R. Morley for helpful suggestions and to the Directors of the Plessey Company for permission to publish this work.

\section{REFERENCES}

1. T. Robertson, "The properties of polypropylene and their influence on the behaviour of polypropylene capacitors", Elect. Comp., October 1972, p. 948.

2. M. J. Schönhuber, "Verlauf der zündspannung von edelund molekülgasen in einer ebenen elektrodenanordnung im grob-, fein- und hochvakuumbersich", Arch. Electrotech., 52, 28 (1968).

3. J. H. Mason, "Dielectric breakdown in solid insulation", Prog. in Dielect., 1, 1 (1959). 
4. T. W. Dakin and D. Berg, "Theory of gas breakdown", Prog. in Dielect., 4, 151 (1962).

5. P. M. Morse and H. Feshbach, "Methods of Theoretical Physics", (McGraw-Hill 1953).

6. E. Nasser, H. Heiszler and M. Abou-Seada, "Field criterion for sustained streamer propagation". J. Appl. Phys., 39, 3707 (1968).
7. J. H. Mason, "Breakdown of insulation by discharges", Proc. I.E.E., 100, 149 (1953).

8. B. Bolton, R. Cooper and K. G. Gupta, "Impulse breakdown of perspex by treeing", Proc. I.E.E., 112, 1215 (1965).

9. M. E. Baird, Electrical Properties of Polymeric Materials, Plastics Institute (1973). 

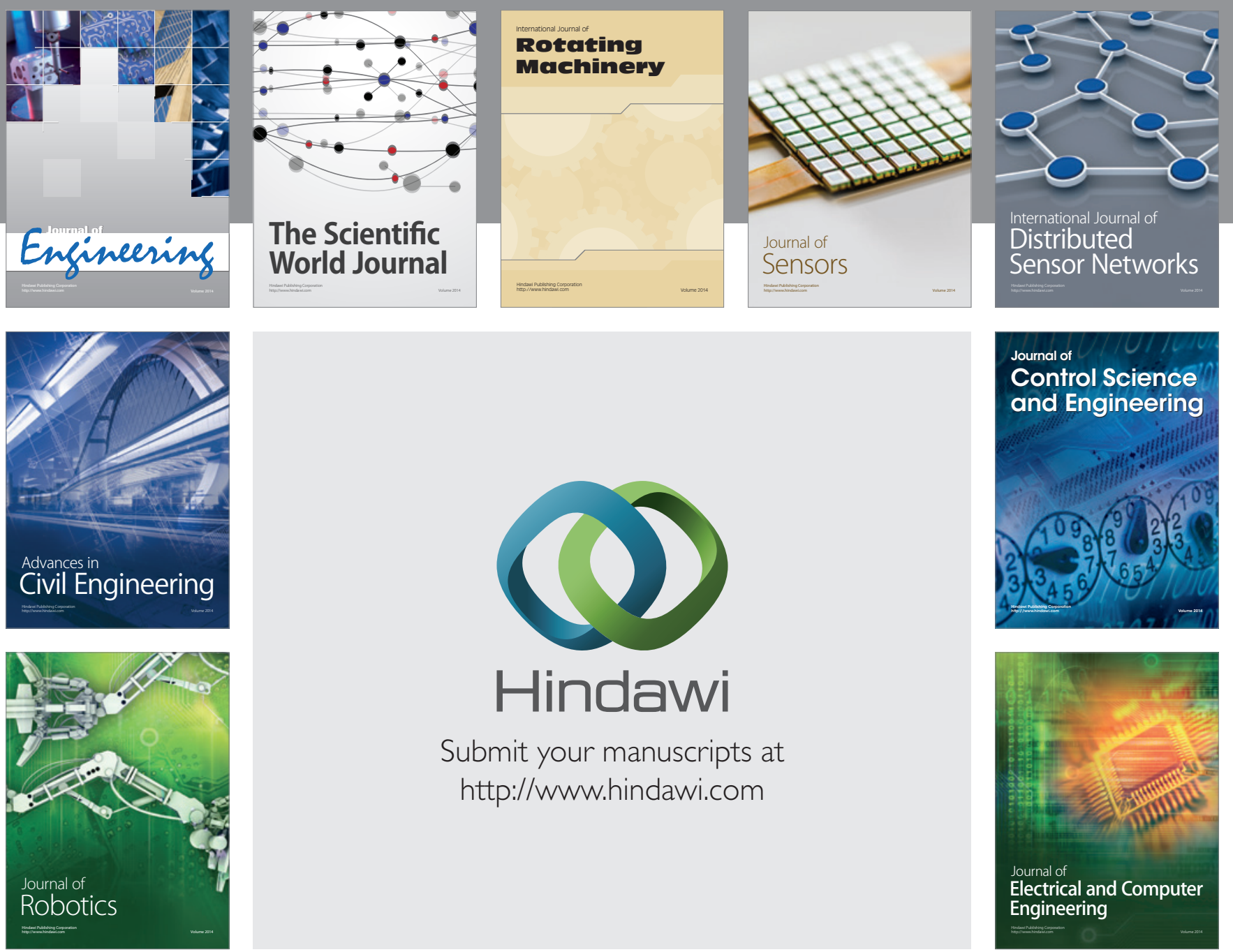

Submit your manuscripts at

http://www.hindawi.com
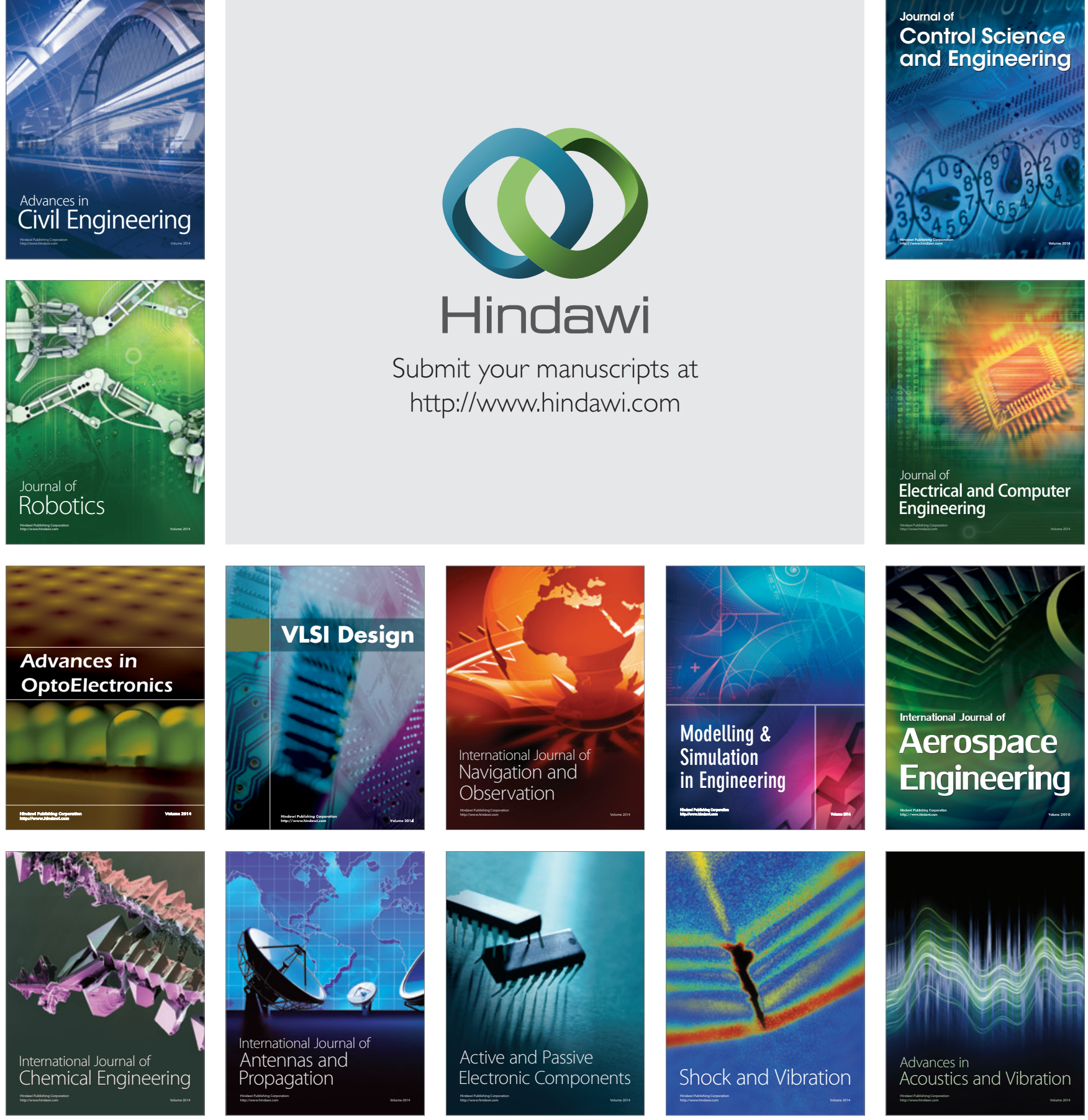